\title{
Methylation of RUNX3 in various types of human cancers and premalignant stages of gastric carcinoma
}

\author{
Tai Young Kim ${ }^{1,2}$, Hyeon Joo Lee ${ }^{2}$, Kyu Sang Hwang ${ }^{2}$, Minjin Lee ${ }^{3}$, Jae Won Kim ${ }^{4}$, \\ Yung-Jue Bang ${ }^{1,2}$ and Gyeong Hoon Kang ${ }^{2,3}$ \\ ${ }^{1}$ National Research Laboratory for Cancer Epigenetics; ${ }^{2}$ Cancer Research Institute; ${ }^{3}$ Department of Pathology \\ and ${ }^{4}$ Department of Obstetrics and Gynecology, Seoul National University College of Medicine, Seoul, South \\ Korea
}

\begin{abstract}
Accumulating evidence has identified a mechanism potentially responsible for the inactivation of tumor suppressor genes, namely transcriptional silencing by aberrant methylation of $\mathrm{CpG}$ islands. A previous study has shown the loss of RUNX3 expression, due to aberrant methylation of its CpG island, in gastric cancer cell lines, suggesting that RUNX3 is a target for epigenetic gene silencing in gastric carcinogenesis. However, there are limited data on the methylation status of RUNX3 in the neoplastic and non-neoplastic tissues in various types of human cancers, including gastric cancer. Here, we report that $60 \%$ of gastric cancer cell lines and $64 \%$ of primary gastric carcinomas $(n=75)$ were methylated at the RUNX3 CpG island. RUNX3 methylation was also detected in hepatocellular carcinomas $(73 \%, n=48)$, larynx cancers $(62 \%, n=37)$, lung cancers $(46 \%, n=24)$, breast cancers $(25 \%, n=25)$, prostate cancers $(23 \%, n=44)$, endometrial cancers $(12.5 \%, n=24)$, colon cancers $(4.9 \%, n=61)$ and uterine cervical cancers $(2.5 \%, n=40)$, showing that $R U N X 3$ methylation is not restricted to gastric cancer. Interestingly, the RUNX3 methylation was especially frequent in tumors from tissues of a foregut derivative, that is, the stomach, liver, larynx and lung. Next, the methylation status of RUNX3 in various nonneoplastic tissues was examined, including the premalignant lesions of gastric carcinomas. The RUNX3 methylation was found in $8.1 \%$ of chronic gastritis $(n=99), 28.1 \%$ of intestinal metaplasia $(n=32), 27.3 \%$ of gastric adenomas $(n=77)$ and $64 \%$ of gastric carcinomas $(n=75)$, but not in chronic hepatitis B, normal prostate and colon mucosa, even though in cases of chronic hepatitis, the methylation frequency of its neoplastic tissues was very high. In conclusion, RUNX3 methylation is frequently found in human cancers, including gastric cancer, and is mostly cancer specific, with the exception of the stomach, and thus, might be useful as a potential diagnostic biomarker of cancer.
\end{abstract}

Laboratory Investigation (2004) 84, 479-484, advance online publication, 16 February 2004; doi:10.1038/labinvest.3700060

Keywords: biomarker; $\mathrm{CpG}$ islands; DNA methylation; gastric cancer; RUNX3

The mammalian RUNX protein is a transcription factor, containing a highly conserved DNA binding domain, designated as a 'runt domain' (RD), which shares a sequence similarity with Drosophila Runt. ${ }^{1}$ Three different mammalian RUNX, termed RUNX1 (also called PEBP2aB/CBFA2/AML1), RUNX2 (PEBP2aA/CBFA1/AML3) and RUNX3 (PEBP2aC/ CBFA3/AML2), have been identified. All three RUNX proteins physically associate with SMAD proteins, downstream targets of the TGF-beta/BMP

Correspondence: GH Kang, MD Department of Pathology, Seoul National University Hospital, 28 Yongon-dong, Chongno-gu, Seoul 110-744, South Korea

E-mail: ghkang@snu.ac.kr

Received 01 August 2003; revised 12 December 2003; accepted 16

December 2003; published online 16 February 2004 signaling, and play important roles in mammalian development. $^{2}$ RUNX1, especially, is required for hematopoiesis, ${ }^{3}$ and its mutations have been observed in about $30 \%$ of human acute leukemia, ${ }^{4}$ whereas RUNX2 is important for bone formation. ${ }^{5} \mathrm{~A}$ recent study revealed that RUNX3 plays an important role in the genesis and progression of human gastric cancer, and functions as a tumor suppressor gene. ${ }^{6}$

Aberrant methylation of promoter $\mathrm{CpG}$ islands, recognized as an alternative to gene mutation or deletion in human cancer, ${ }^{7}$ is associated with transcriptional silencing of several genes, such as p16, ${ }^{8}$ hMLH1, ${ }^{9}$ RASSF $1 A^{10}$ and E-cadherin, ${ }^{11}$ in human cancer. Li et al. ${ }^{6}$ showed the relationship of the loss of RUNX3 expression, in part, with aberrant methylation of its $\mathrm{CpG}$ island, in gastric cancer cell 
lines, suggesting that RUNX3 is a target for epigenetic gene silencing in gastric carcinogenesis. However, they did not examine the methylation status of RUNX3 in the premalignant stages of gastric carcinomas. If the aberrant methylation of $R U N X 3$ contributes to tumor initiation, the methylation change would be expected to be found in the premalignant stages of gastric carcinomas. Another expectation is that the aberrant methylation of RUNX3 might be found in human cancers of other tissue types if RUNX3 serves as a tumor suppressor gene. At present, it is unknown whether RUNX3 methylation is restricted to gastric cancer or if it is a common event in human cancers of other tissue types.

In the present study, the methylation status of $R U N X 3$ was studied in gastric carcinomas, their premalignant stages (chronic gastritis, intestinal metaplasia and gastric adenoma) and various types of human cancers. In addition, a topographic analysis of $R U N X 3$ methylation was attempted in a stomach containing a gastric carcinoma.

\section{Materials and methods}

\section{Tumor Samples and Cell Lines}

Formalin-fixed, paraffin-embedded archival tissues of gastric carcinomas $(n=74)$, gastric adenomas $(n=77)$, chronic gastritis with/without intestinal metaplasia ( $n=32$ and 99, respectively), hepatocellular carcinomas $(n=48)$, lung cancers $(n=24)$, larynx cancers $(n=37)$, colon cancers $(n=61)$, breast cancers $(n=25)$, prostate cancers $(n=44)$, endometrial cancers $(n=24)$ and uterine cervical cancers $(n=40)$ were obtained from the files of the Department of Pathology, Seoul National University Hospital, Seoul, Korea. Through light microscopic examination of the hematoxylin-eosin-stained slides, tissue blocks were selected for having tumor cells constituting more than $30 \%$ of their tumor volumes. Five gastric cancer cell lines (SNU-5, -484, $-601,-620$, and -719) were obtained from the Korean Cell Line Bank (Seoul), and were maintained in RPMI-1640 medium, supplemented with $10 \%$ fetal bovine serum and gentamycin $(10 \mu \mathrm{g} / \mathrm{ml})$, at $37^{\circ} \mathrm{C}$ in a humidified $5 \% \mathrm{CO}_{2}$ atmosphere.

\section{Topographic Analysis}

Multiple tissue samples, from two surgically excised gastric cancers, were collected, snap-frozen and stored at $-80^{\circ} \mathrm{C}$, until processed. The gastrectomy specimens were photographed and traced on a $2 \mathrm{~cm}$ grid, with the sites of biopsy marked to preserve the topographic relationship between biopsies. The samples were taken at $3 \mathrm{~cm}$ intervals along the longitudinal axis and $1.5 \mathrm{~cm}$ intervals along the vertical axis, from both the tumoral and nontumoral areas throughout the stomach.

\section{DNA Preparation}

The histologic sections, of $20 \mu \mathrm{m}$ thickness, were dewaxed in xylene, with subsequent ethanol treatment. Genomic DNA was isolated by the standard method of proteinase $\mathrm{K}$ digestion and phenolchloroform/isoamylalcohol extraction.

\section{Sodium Bisulfite Treatment and MSP}

Both the normal and tumor DNAs were subjected to sodium bisulfite modification, as described previously. ${ }^{12}$ Briefly, $5 \mu \mathrm{g}$ of DNA was denatured with $2 \mathrm{M} \mathrm{NaOH}$, followed by treatment with $1 \mathrm{mM}$ hydroquinon and $3.5 \mathrm{M}$ sodium sulfite, with incubation for $16 \mathrm{~h}$ at $55^{\circ} \mathrm{C}$. Following purification, using a JETSORB gel extraction kit (Genomed, Oeynhausen, Germany), the DNA was treated with $3 \mathrm{M} \mathrm{NaOH}$, and precipitated with three volumes of $100 \%$ ethanol and a one-third volume of $7.5 \mathrm{M} \mathrm{NH}_{4} \mathrm{Ac}$ at $-20^{\circ} \mathrm{C}$. The precipitated DNA was washed with $70 \%$ ethanol and dissolved in distilled water. The sodium bisulfite-modified DNA was subject to MSP using primer sets: methylated reactions, 5'-TAT TCG TTA GGG TTC GTT CGT-3' (sense) and $5^{\prime}$-AAA CAA CCA CGA AAA ACG AC-3' (antisense) and unmethylated reactions, 5'-AAG TGG GAA AGT AGA AGT GGT G - $3^{\prime}$ (antisense) and $5^{\prime}$-CCA AAC AAA CTA CAA ACA ACC A-3' (antisense). The $25 \mu \mathrm{l}$ total reaction volume contained a PCR mixture of $1 \mathrm{X}$ PCR buffer $(16.6 \mathrm{mM}$ $\left(\mathrm{NH}_{4}\right)_{2} \mathrm{SO}_{4} / 67 \mathrm{mM}$ Tris/pH 8.8/6.7 $\mathrm{mM} \mathrm{MgCl}{ }_{2} / 10 \mathrm{mM}$ $\beta$-mercaptoethanol), dNTPs (each at $1 \mathrm{mM}$ ), primers (10 pmol each) and bisulfite-modified DNA (30-50 ng). The reactions were hot-started at $97^{\circ} \mathrm{C}$ for $5 \mathrm{~min}$, prior to the addition of 0.75 units of Taq polymerase (Takara Shuzo Co., Kyoto, Japan). The PCR conditions were as follows: 35 cycles of denaturation at $95^{\circ} \mathrm{C}$ for $40 \mathrm{~s}$, annealing at $60^{\circ} \mathrm{C}$ for $50 \mathrm{~s}$, and finally a $50 \mathrm{~s}$-extension at $72^{\circ} \mathrm{C}$. A final 10-min extension at $72^{\circ} \mathrm{C}$ completed each PCR. Each set of PCR reaction products was loaded onto $2.5 \%$ agarose gels, ethidium bromide-stained and visualized under UV light. Samples showing signals approximately equivalent to that of the size marker $(7 \mathrm{ng} / \mu \mathrm{l})$ were scored as methylated. Samples giving faint positive signals were repeated three times and only those samples with consistent positive signals were scored as methylated.

\section{Bisulfite Genomic Sequencing}

For bisulfite sequencing of five gastric cell lines, sodium bisulfite-treated genomic DNA was amplified using the following primers: $5^{\prime}$-ATT TTG GAG GAT TTG TTT TGG G-3' (sense) and 5'-CAA CCT ACC CRA CTA ATC CC-3' (antisense). The conditions of the amplification were as follows: $95^{\circ} \mathrm{C}$ for $12 \mathrm{~min}$, and 35 cycles of $95^{\circ} \mathrm{C}$ for $30 \mathrm{~s}, 50^{\circ} \mathrm{C}$ for $30 \mathrm{~s}$, and $72^{\circ} \mathrm{C}$ for $30 \mathrm{~s}$, followed by final extension at 
$72^{\circ} \mathrm{C}$ for $10 \mathrm{~min}$. PCR reaction products were gelpurified and cloned into pCR2.1-TOPO vector (Invitrogen, Carlsbad, CA, USA). The inserted PCR fragments of individual clones were sequenced using an ABI Prism Dye Terminator Cycle Sequencing Kits (Perkin-Elmer, Foster City, CA, USA) and ABI Prism 377 DNA Sequencer (Perkin-Elmer).

\section{Drug Treatment and RT-PCR}

The cells were seeded at a density of $1 \times 10^{6}$ cells/ $100 \mathrm{~mm}$ dish, and treated $24 \mathrm{~h}$ later with $5 \mu \mathrm{M}$ of 5 aza-dC for 4 days. At the end of the treatment, the medium was removed and the RNA was isolated. cDNA was synthesized using M-MLV reverse transcriptase (Invitrogen), with random hexamers, following the manufacturer's instructions, and then amplified, with primers specific for the RUNX3 gene; $5^{\prime}$-GAG TTT CAC CCT GAC CAT CAC TGT G$3^{\prime}$ (sense) and 5'-GCC CAT CAC TGG TCT TGA AGG TTG T-3' (antisense). The PCR reactions were performed as follows: $95^{\circ} \mathrm{C}$ for $5 \mathrm{~min}$, and 35 cycles of $95^{\circ} \mathrm{C}$ for $30 \mathrm{~s}, 55^{\circ} \mathrm{C}$ for $30 \mathrm{~s}$ and $72^{\circ} \mathrm{C}$ for $30 \mathrm{~s}$, followed by incubation at $72^{\circ} \mathrm{C}$ for $10 \mathrm{~min}$. $\beta$-actin was amplified to estimate the efficiency of cDNA synthesis as described previously. ${ }^{13}$ The PCR products were resolved on $1 \%$ agarose gels.

\section{Results}

Methylation-specific PCR (MSP) was performed to analyze the methylation status of the RUNX3 CpG islands in gastric cancer cell lines. SNU-5 and SNU484 cells were unmethylated at the RUNX3 CpG islands, whereas SNU-601, SNU-620 and SNU-719 cells were fully methylated at this locus (Figure 1a). To verify MSP data and examine the density of methylated CpG sites, we performed bisulfite genomic sequencing on DNA from all cell lines. The promoter region spanning $30 \mathrm{CpG}$ sites was amplified by PCR using sodium bisulfite-modified DNA as templates, and 3-5 PCR clones of each cell line were sequenced. As shown in Figure 1b, SNU-5 and SNU484 cells were almost completely unmethylated at the $\mathrm{CpG}$ sites in the clones analyzed, whereas SNU601, SNU-620 and SNU-719 cells showed the complete methylation pattern.

RUNX3 mRNA expression in these cell lines was evaluated by reverse-transcription PCR (RT-PCR). $R U N X 3$ transcripts were observed in the unmethylated cell lines, but were not detected in any of the methylated cell lines (Figure 1c). To determine whether DNA methylation might be involved in the loss of RUNX3 mRNA expression, three methylated cell lines (SNU-601, SNU-620 and SNU-719) were treated with the demethylating agent, 5-aza2 'deoxycytidine (5-aza-dC). Restoration of RUNX3 mRNA expression was observed in all the methylated cell lines after treatment with 5-aza-dC, confirming that the loss of RNUX3 mRNA expres- sion was related to the aberrant methylation of its CpG islands (Figure 1c).

Next, the RUNX3 methylation was examined to observe if it occurs in primary gastric cancer and various other types of human cancer. In all, $64 \%$ of primary gastric carcinomas $(n=75)$ were found to be methylated at the RUNX3 CpG island, showing a frequency similar to those in a previous study ${ }^{19}$ (Figure 2). The RUNX3 methylation was also frequently detected in hepatocellular carcinomas $(73 \%, n=48)$, larynx cancers $(62 \%, n=37)$, lung cancers $(46 \%, n=24)$, breast cancers $(25 \%, n=25)$, prostate cancers $(23 \%, n=44)$ and endometrial cancers $(12.5 \%, n=24)$, but was rarely detected in colon cancers $(4.9 \%, n=61)$ or uterine cervical cancers $(2.5 \%, n=40)$. These results show that

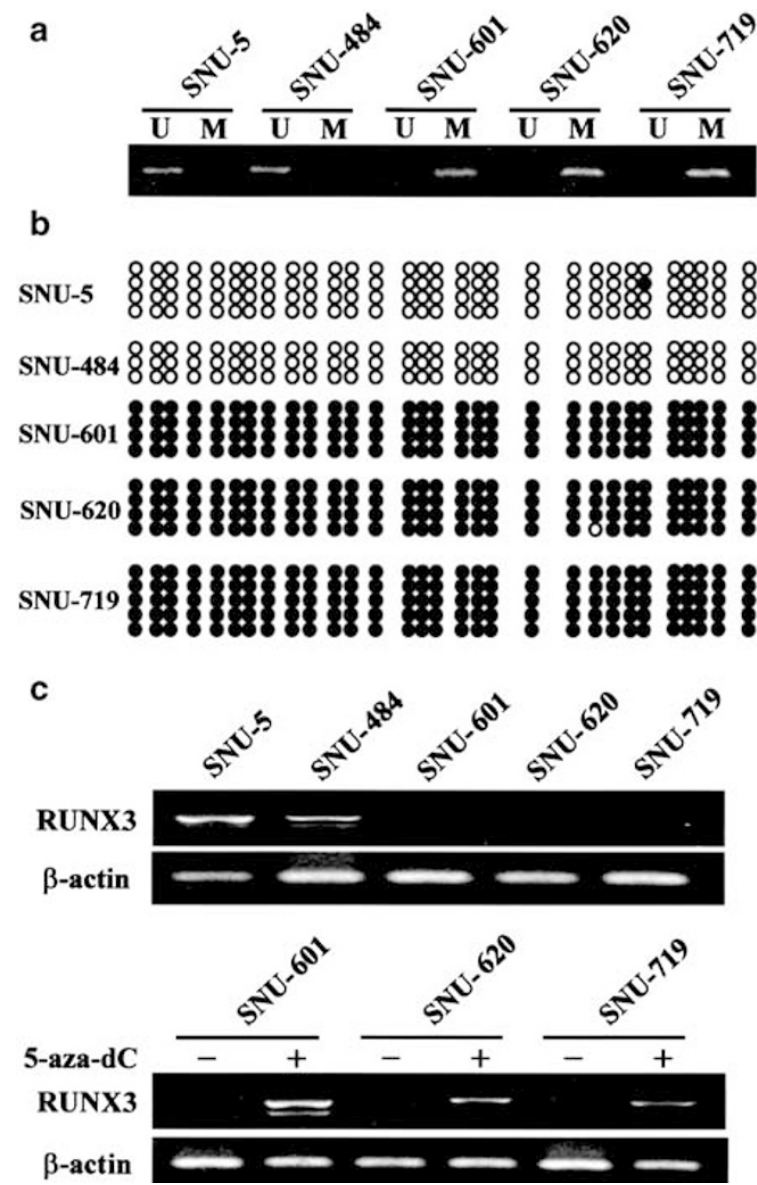

Figure 1 RUNX3 methylation in gastric cancer cell lines. (a) The presence of a visible PCR product in lane U indicates the presence of unmethylated alleles; the presence of a PCR product in lane M indicated the presence of methylated alleles. (b) Bisulfite genomic sequencing of the RUNX3 CpG island was performed in gastric cell lines. Each circle indicates a $\mathrm{CpG}$ site and each line of circles represents the analysis of a single cloned allele. $\bigcirc$, an unmethylated $\mathrm{CpG}$ site; $\boldsymbol{0}$, a methylated $\mathrm{CpG}$ site. (c) The expression of RUNX3 mRNA was determined in gastric cancer cell lines by RT-PCR. The loss of RUNX3 mRNA expression was found in three of five cell lines, but not in SNU-5 and SNU-484 cell lines. Treatment with 5 -aza-dC of the nonexpressing gastric cancer cell lines containing methylated alleles restored the RUNX3 mRNA expression. 
RUNX3 methylation is not restricted to gastric cancer.

Accumulating evidence indicates that hypermethylation of $\mathrm{CpG}$ islands begins early in cancer progression, and in some cases, may precede the neoplastic process. If the aberrant methylation of certain genes contributes to tumor initiation, the methylation change would be expected to be found in premalignant lesions. To evaluate this possibility, the methylation status of $R U N X 3$ in premalignant lesions of gastric carcinomas, including chronic gastritis, intestinal metaplasia and gastric adenomas, was examined. RUNX3 methylation was found in $8.1 \%$ of chronic gastritis $(n=99), 28.1 \%$ of intestinal metaplasia $(n=32), 27.3 \%$ of gastric adenomas $(n=77)$ and $64 \%$ of gastric carcinomas $(n=75)$. The difference in the methylation frequency was significant between chronic gastritis and intestinal metaplasia $(P=0.006)$, and between intestinal metaplasia and gastric carcinomas $(P=0.001)$. In addition, chronic liver disease (chronic hepatitis B) $(n=40)$, normal prostate tissue $(n=24)$ and normal colon tissue $(n=60)$ were

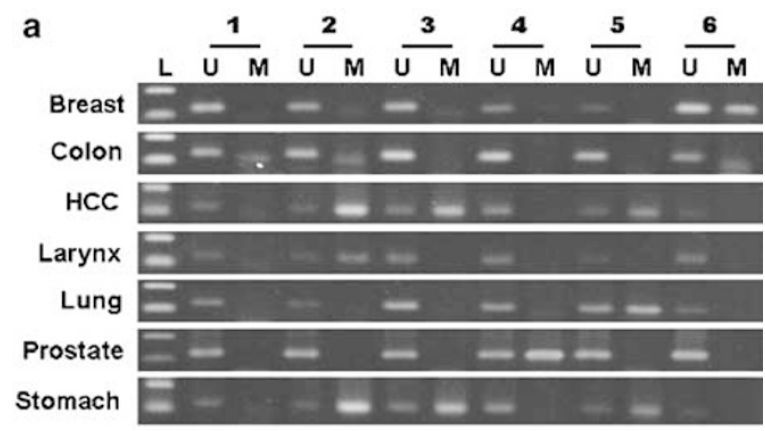

b

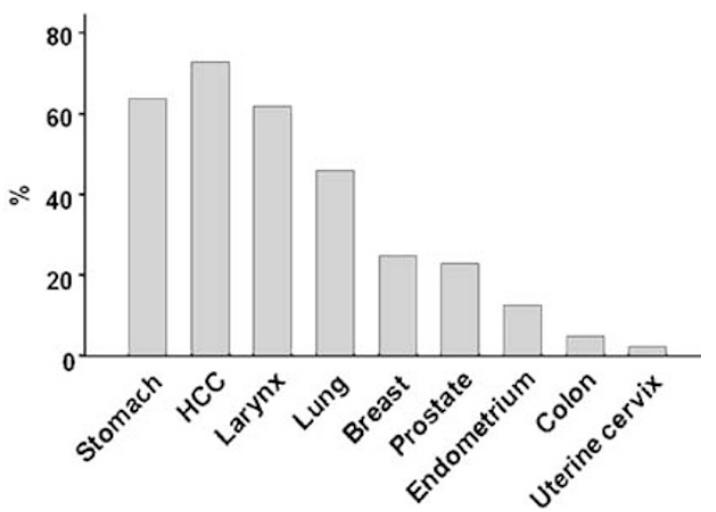

Figure 2 RUNX3 methylation across tumor types. (a) Representative samples of MSP analysis. Products that were amplified with primers specific to the unmethylated or methylated alleles of $R U N X 3$, following bisulfite modification, were loaded onto $2.5 \%$ agarose gels. L, 100-bp DNA ladder. (b) Frequency of RUNX3 methylation. RUNX3 methylation was frequently detected in gastric cancers $(64 \%, n=75)$, hepatocellular carcinomas $(73 \%$, $n=48)$, larynx cancers $(62 \%, n=37)$, lung cancers $(46 \%, n=24)$, breast cancers $(25 \%, n=25)$, prostate cancers $(23 \%, n=44)$ and endometrial cancers $(12.5 \%, n=24)$, but was rarely detected in colon cancers $(4.9 \%, n=61)$ or uterine cervical cancers $(2.5 \%$, $n=40$ ). analyzed. However, RUNX3 methylation was not found in these tissues.

The spatial distribution of $R U N X 3$ methylation was determined by MSP assays of multiple samples obtained from the topographic analysis of two cases of surgically resected gastric carcinoma (case 1, 53year-old male; case 2, 42-year-old male). The results are depicted in Figure 3, which clearly demonstrates that RUNX3 methylation was confined to the tumoral portion. One of 29 nontumoral samples harbored RUNX3 methylation in case 2, but none of the nontumoral samples showed RUNX3 methylation in case 1.

\section{Discussion}

Transcriptional silencing of tumor suppressor genes by aberrant methylation of $\mathrm{CpG}$ islands plays a crucial role in the development of various cancers. Many genes involved in the regulation of the cell cycle, tissue invasion, DNA repair and apoptosis have been shown to be inactivated by this type of epigenetic mechanism. Recently, the loss of RUNX3 expression, accompanied by the aberrant methylation of its promoter, was reported in gastric cancer cell lines. Consistent with these data, our results from MSP and RT-PCR showed the relation between the RUNX3 methylation status and its expression in gastric cancer cell lines.

In addition, whether RUNX3 methylation occurs in primary gastric cancer and various types of human cancers was examined. The results of this study show that RUNX3 methylation is not restricted to gastric cancer, but also occurs frequently in other types of cancer, such as liver, larynx, lung, breast, prostate and endometrial cancers, suggesting that the RUNX3 methylation constitutes a common feature of many cancers. However, it is necessary to confirm that RUNX3 methylation is representative of the loss of its expression in other types of cancer. RUNX3 genes can be transcribed from two alternative promoters, P1 (distal) and P2 (proximal), and therefore, expressed through a $R U N X 3 \mathrm{P} 1$ promoter, even though P2 promoter was methylated. ${ }^{14,15}$ Contrary to the ubiquitously active P2 promoter, the activity of the P1 promoter is restricted to a few tissue types, such as the thymus and ovary, and there are no data for the activity of the P1 promoter in the various tissues examined. ${ }^{15}$

It is important to note that loss of heterozygosity (LOH) at $1 \mathrm{p} 36.1$, where the RUNX3 gene locates, was detected in the most hypermethylated cases of testicular yolk sac tumors from infants. ${ }^{16}$ Therefore, RUNX3 seems to follow the two-hit model of inactivation of tumor suppressor gene, namely loss of one allele and inactivation of the other by the hypermethylation. Further study on the $\mathrm{LOH}$ in various tumor tissues is also needed to better understand the molecular mechanism for the loss of RUNX3 expression. 
a
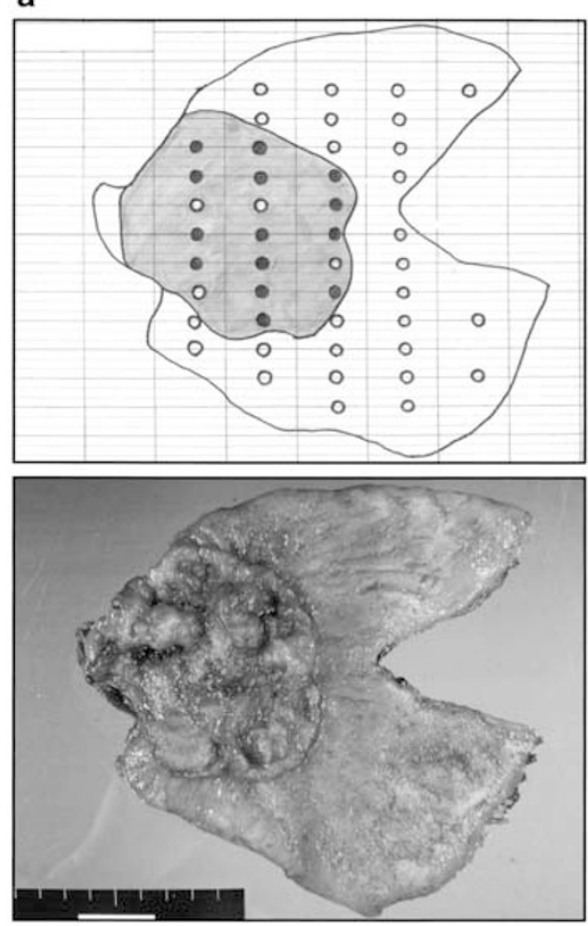

b

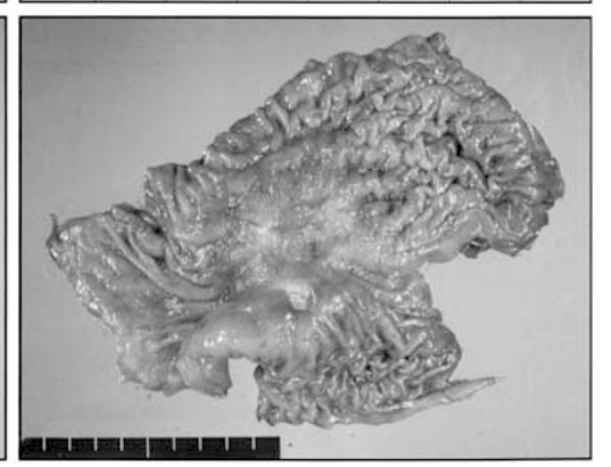

Figure 3 Topographic analysis of $R U N X 3$ methylation. Two gastrectomy specimens (a, case 1; b, case 2) are illustrated, with each circle representing the biopsy sites. Open circle, $\bigcirc$ and closed circle, $\bigcirc$ indicate samples with no RUNX3 methylation and with $R U N X 3$ methylation, respectively. Tumoral portions are shown in gray. Matched photographs of the gross specimen are given in the lower row.

In recent years, several attempts have been made to examine the methylation status of specific genes in the premalignant stages of cancer. ${ }^{17,18}$ In this study, it was found that RUNX3 methylation occurs in chronic gastritis, the early stages of multistep gastric carcinogenesis, although the methylation frequency $(8.1 \%)$ was lower than those of intestinal metaplasia $(28.1 \%)$ or gastric adenomas $(27.3 \%)$. The methylation frequency of $R U N X 3$ was much higher in gastric carcinomas (64\%) than those in premalignant lesions. This result suggests that RUNX3 methylation increases with the progression of the lesion along the multistep gastric carcinogenesis, and is related to the genesis of gastric carcinomas. Recently, Waki et al ${ }^{19}$ reported that $8 \%$ of non-neoplastic gastric mucosa, from patients without gastric cancer, harbored the RUNX3 methylation, which were seen only in patients aged over 77 years. In the present study, RUNX3 methylation was found in non-neoplastic gastric mucosa, from patients without gastric cancer, aged younger than 50 years $(3.2 \%$, two of 62$)$, and with methylation frequencies of $17.9,20$ and $13.6 \%$ in patients in their 6th, 7th and 8th decades, respectively, exhibiting an increase in the methylation with aging. In contrast to the non-neoplastic gastric mucosa, RUNX3 methylation was not found in other nonneoplastic tissues, such as chronic hepatitis B, prostate and colon mucosa, even though in hepatitis, the methylation frequency of its neoplastic tissues was very high. Taken together, with the exception of the stomach, RUNX3 methylation was very rare in non-neoplastic tissues, so the methylation can be regarded as a cancer-specific change. In the stomach, RUNX3 methylation is cancer-related, but can also occur in non-neoplastic gastric mucosa.

When the methylation frequency of RUNX3 was compared between different tissue types of human cancers, an interesting trend was observed; high frequencies of RUNX3 methylation were seen in tumors from tissues of a foregut derivative, that is, the stomach, liver, larynx and lung. In Esteller et al's study, ${ }^{20}$ which investigated the methylation pattern of human cancers of multiple tissue types, the methylation patterns of gastric cancer were similar to those of colon cancer. However, the RUNX3 methylation showed the contrast between gastric cancer and colon cancer.

In conclusion, RUNX3 methylation was frequently found in human cancers, including gastric cancer, and was mostly cancer specific, with the exception of the stomach, and thus might be useful as a potential diagnostic biomarker of cancer.

\section{Acknowledgement}

This work was supported in part by R02-200200040-0 from the Basic Research Program of the Korea Science and Engineering Foundation, by the Ministry of Science \& Technology of Korea through the National Research Laboratory Program for 
Cancer Epigenetics, and by the BK21 project for Medicine, Dentistry, and Pharmacy, Seoul, Korea.

\section{References}

1 Gergen JP, Butler BA. Isolation of the Drosophila segmentation gene runt and analysis of its expression during embryogenesis. Genes Dev 1988;2: 1179-1193.

2 Hanai J, Chen LF, Kanno T, et al. Interaction and functional cooperation of PEBP2/CBF with Smads. Synergistic induction of the immunoglobulin germline Calpha promoter. J Biol Chem 1999;274: 31577-31582.

3 Okuda T, van Dursen J, Hiebert SW, et al. AML1, the target of multiple chromosomal translocation in human leukemia, is essential for normal fetal liver hematopoiesis. Cell 1996;84:321-330.

4 Look AT. Oncogenic transcription factors in the human acute leukemias. Science 1997;278:1059-1064.

5 Lee B, Thirunavukkarasu K, Zhou L, et al. Missense mutations abolishing DNA binding of the osteoblastspecific transcription factor OSF2/CBFA1 in cleidocranial dysplasia. Nat Genet 1997;16:307-310.

6 Li QL, Ito K, Sakakura C, et al. Causal relationship between the loss of RUNX3 expression and gastric cancer. Cell 2002;109:113-124.

7 Momparler RL, Bovenzi V. DNA methylation and cancer. J Cell Physiol 2000;183:145-154.

8 Merlo A, Herman JG, Mao L, et al. 5' CpG island methylation is associated with transcriptional silencing of the tumour suppressor p16/CDKN2/MTS1 in human cancers. Nat Med 1995;1:686-692.

9 Kane MF, Loda M, Gaida GM, et al. Methylation of the hMLH1 promoter correlates with lack of expression of hMLH1 in sporadic colon tumors and mismatch repairdefective human tumor cell lines. Cancer Res 1997;57: 808-811.
10 Dammann R, Li C, Yoon JH, et al. Epigenetic inactivation of a RAS association domain family protein from the lung tumour suppressor locus 3p21.3. Nat Genet 2000;25:315-319.

11 Yoshiura K, Kanai Y, Ochiai A, et al. Silencing of the E-cadherin invasion-suppressor gene by CpG methylation in human carcinomas. Proc Natl Acad Sci USA 1995;92:7416-7419.

12 Herman JG, Graff JR, Myohanen S, et al. Methylationspecific PCR: a novel PCR assay for methylation status of CpG islands. Proc Natl Acad Sci USA 1996;93: 9821-9826.

13 Jong HS, Park YI, Kim S, Sohn JH, Kang SH, Song SH, et al. Up-regulation of human telomerase catalytic subunit during gastric carcinogenesis. Cancer 1999;86: 559-565.

14 Bangsow C, Rubins N, Glusman G, et al. The RUNX3 gene-sequence, structure and regulated expression. Gene 2001;279:221-232.

15 Rini D, Calabi F. Identification and comparative analysis of a second runx3 promoter. Gene 2001;273: 13-22.

16 Kato N, Tamura G, Fukase M, et al. Hypermethylation of the RUNX3 gene promoter in testicular yolk sac tumor of infants. Am J Pathol 2003;163:387-391.

17 Kang GH, Lee S, Kim JS, et al. Profile of aberrant CpG island methylation along multistep gastric carcinogenesis. Lab Invest 2003;83:519-526.

18 Cho B, Lee H, Jeong S, et al. Promoter hypomethylation of a novel cancer/testis antigen gene CAGE is correlated with its aberrant expression and is seen in premalignant stage of gastric carcinoma. Biochem Biophys Res Commun 2003;307:52-63.

19 Waki T, Tamura G, Sato M, et al. Promoter methylation status of DAP-kinase and RUNX3 genes in neoplastic and non-neoplastic gastric epithelia. Cancer Sci 2003; 94:360-364.

20 Esteller M. CpG island hypermethylation and tumor suppressor genes: a booming present, a brighter future. Oncogene 2002;21:5427-5440. 